\title{
Producción de Jarabes Edulcorantes por Hidrólisis Enzimática del Almidón de Name Variedad (Dioscorea rotundata)
}

\section{Sweetening syrup production by enzymatic hydrolysis of starch variety yam (Dioscorea rotundata)}

\author{
Vidal Tovar Carlos Ramón', Páez Gisela²
}

${ }^{1}$ Escuela de Ciencias Básicas Tecnología e Ingeniería, Universidad Nacional Abierta y a Distancia, Cead Valledupar, Colombia. carvit67@yahoo.es

2 Departamento de Ingeniería Bioquímica, Escuela de Ingeniería Química, Facultad de Ingeniería, Universidad del Zulia, Venezuela. gpaezr@gmail.com

\section{Resumen}

En el proceso de obtención de jarabes edulcorantes por hidrólisis enzimática del almidón de ñame espino (Dioscorea rotundata), el almidón fue extraído por medio de un rayado, lavado, sedimentado y posterior secado. Se cuantificó el rendimiento teniendo en cuenta la cantidad de materia prima inicial y se le determinó la concentración de almidón, amilosa, amilopectina, fibra cruda, cenizas, proteína, grasas y humedad acorde con lo exigido en las normas AOAC, COVENIN e ICONTEC. La hidrólisis enzimática del almidón se realizó aplicando $\partial$ - amilasa, glucoamilasa y pululanasa en soluciones de almidón al 36 y 46\% p/p variando el orden de aplicación de la glucoamilasa y pululanasa; se determinó el pH, grados Brix, humedad, azúcares reductores (AR), azúcares totales (AT) y el equivalente dextrosa (ED) en los jarabes obtenidos. En la licuefacción se obtuvieron jarabes edulcorantes intermedios con un ED del $18,81 \%$ y el $22,15 \%$. Jarabes de baja y media conversión con un ED entre el 34-45\% en la primera sacarificación y jarabes de alta conversión con un ED entre el 75-79 $\%$ como producto final. Los valores anteriores permiten la utilización del almidón de ñame espino para la producción de jarabes de múltiples usos en diferentes procesos de la industria agroalimentaria.

Palabras clave: ñame, jarabes edulcorantes, hidrólisis enzimática, equivalente dextrosa.

\section{Abstract}

Sweeteners syrups produced by enzymatic hydrolysis from starch of hawthorn yam (Dioscorea rotundata). The starch was extracted by a scratched, washed, sedimented and drying; the yield was quantified taking into account the amount of initial raw material and was determined the concentration of starch, amylose, amylopectin, crude fiber, ash, protein, fat and humidity in accordance with the requirements of the AOAC standards, and ICONTEC COVENIN. Enzymatic hydrolysis of starch was conducted using $\partial$-amylase, glucoamylase and pullulanase in starch solutions at 36 and $46 \% \mathrm{w} / \mathrm{w}$ varying the order of application of glucoamylase and pullulanase were determined $\mathrm{pH}$, Brix, moisture, reducing sugars (AR), total sugar (TS) and the dextrose equivalent (ED) in the syrups obtained. In the liquefaction were obtained with an intermediate syrups sweeteners ED $18.81 \%$ and $22.15 \%$. Syrups low and medium conversion with an ED between $34-45 \%$ in the first saccharification and high conversion syrups with a DE between $75-79 \%$ as a final product. The above values allow the use of hawthorn yam starch syrup production for multiple uses in different food industry processes.

Key words: yam, syrups, enzymatic hydrolysis, dextrose equivalent. 


\section{Introducción}

El ñame es una planta tropical de origen africano y asiático perteneciente al orden Dioscoreales, familia Dioscoreáceas, contiene 6 géneros pero Dioscorea es el más importante con 600 especies identificadas y solo 12 especies comestibles. Las especies más cultivadas corresponden a Dioscorea alata, $D$. rotundata, $D$. cayennensis, $D$. esculenta, $D$. bulb ífera y $D$. trífida; la primera es la preferida en la producción de tubérculos para el consumo humano. [1]. El área mundial cultivada es de 2.933.000 hectáreas repartidas en 3 regiones principales: África Occidental, sur de Asia incluyendo parte de China, Japón, Oceanía y los países del Caribe, estas tienen una producción de 28.126.000 toneladas, siendo el mayor productor el continente Africano con un 95,3\% del total de la producción mundial [2].

En América, el ñame es importante en Brasil, Colombia, Haití, Venezuela y Antillas Francesas por ser un cultivo de autoconsumo por parte de la población campesina. En Colombia se pueden encontrar varias especies de ñame como el ñame criollo ( $D$. alata), ñame espino ( $D$. rotundata), ñame papa $(D$. bulbífera), ñame azúcar $(D$. esculenta) y ñampin ( $D$. trífida). Se considera que $D$. alata y $D$. rotundata son las especies de mayor importancia tanto por el área sembrada como por la demanda del tubérculo, seguidas por $D$. trífida. De la producción total de ñame en Colombia, el $17,7 \%$ se destina al autoconsumo, el $4,2 \%$ para semilla y el $78,1 \%$ se dirigen al mercado en fresco. No es frecuente el uso en alimentación animal y actualmente los procesos de transformación no se realizan a escala industrial [1].

Una de las materias primas de mayor utilidad en los procesos industriales de alimentos son los agentes edulcorantes. En general, este tipo de industria utiliza en su proceso de producción diversas materias primas edulcorantes, como el azúcar común o sacarosa, glucosa, lactosa, fructuosa, jarabes u otro agente que produzca la sensación de dulzor agradable al paladar en los productos listos para consumir. Algunos productos de alta concentración de sólidos, como mermeladas, arequipes o dulces pueden llegar a utilizar hasta un $45 \%$ en su formulación, con el fin de conservar el producto o proporcionar las características organolépticas de cada producto.

A partir de 1970, por el elevado precio del mercado internacional de estos azúcares, la investigación se ha enfocado en la generación y desarrollo de alternativas de agentes endulzantes o también denominados sustitutos del azúcar; entre estos se encuentran la panela, edulcorantes naturales como el jarabe de maíz rico en fructosa, edulcorantes artificiales como el aspartame (Nutrasweet), la sacarina, la miel y la estevia. Los los jarabes procedentes de almidones de maíz son los de mayor utilidad y comercialización mundial, estos se han posesionado del mercado por el alto costo que tiene el azúcar para la elaboración de un producto. [3].

La hidrólisis ácida o enzimática del almidón permite obtener innumerables productos, entre los cuales se encuentran los jarabes y dextrinas, esto depende de la disponibilidad del almidón en la materia prima y el contenido de amilosa y amilopectina. Entre las diferentes materias primas el ñame se presenta como una alternativa de utilización frente al maíz, yuca, plátano o papa por el contenido de almidón presente en este. En ese sentido, el objetivo principal de este trabajo fue evaluar la producción de jarabes edulcorantes por hidrólisis enzimática del almidón de ñame variedad Dioscorea rotundata para su utilización en la industria de alimentos, lo que permitirá establecer una pauta para el aprovechamiento del ñame como materia prima no convencional en la producción de jarabes edulcorantes. 


\section{Metodología Experimental}

\section{Producción del almidón de ñame (Dioscorea rotundata)}

A partir de $25 \mathrm{~kg}$ de ñame espino (Dioscorea rotundata) obtenido en diferentes puntos del mercado de Bazurto de Cartagena de Indias-Colombia, se procedió a la extracción del almidón por medio del procedimiento aplicado por CORPOICA (2003) así:

- Los rizomas de ñame se lavaron con abundante agua fresca, se eliminaron los restos de impurezas como barro, tierra y raicillas adheridas a la cáscara, se cortaron en segmentos para reducir su tamaño, facilitar su manipulación y completo lavado. Cada uno de los segmentos se troceó, se peló y se lavó de forma inmediata.

- $\quad$ Los trozos de ñame, descascarado y limpios, se rallaron manualmente con un rallador casero; a la pasta obtenida se le adicionó agua en proporción de 1:10 en un tanque, se mezcló completamente por 5 minutos y se dejó por 2 horas en reposo. Al completar la sedimentación de la mezcla fibra y almidón, se descartó el $70 \%$ del contenido de agua con la precaución de no agitar el sedimento contenido en el tanque, el cual estaba compuesto por agua, fibra y almidón, luego se sometió a un doble tamizado con coladores de uso común y tela tipo paño respectivamente. Se descartó la fibra separada y la lechada de almidón obtenida, se le adicionó agua en proporción de 1:10, se mezcló por 5 minutos y se sometió a sedimentación por 1 hora, se le retiró la máxima cantidad de agua posible teniendo el cuidado de no dejar escapar parte de la lechada de almidón. La lechada obtenida se repartió en bandejas plástica y se sometió a secado a $45^{\circ} \mathrm{C}$, con mezclados sucesivos hasta alcanzar un $10 \%$ de humedad en un tiempo de 12 horas.

El almidón obtenido se dejó a temperatura ambiente cubierto con un paño por 6 horas, se empacó en bolsas de polietileno de sello hermético, se almacenó y se caracterizó determinando la concentración de almidón por 2 métodos: el descrito por [4] y una modificación y adaptación de la norma [5].

La Humedad se determinó por la norma [6]; método [7]. La Amilosa y Amilopectina por colorimetría, utilizado por [8], a través de dimetilsul- fòxido (DMSO) y una recta patrón de amilosa preestablecida. Fibra cruda por el método [9].

Las Cenizas fueron determinadas por incineración directa, utilizando el método [9]. Para Proteínas, se utilizó el método de Biuret y Grasas se determinó por el método de soxhlet, sistema tecator A.O.A.C 31.4.02. El pH por normas [10], método [9].

\section{Hidrólisis Enzimática del Almidón}

\section{Enzimas utilizadas}

Las enzimas utilizadas para desarrollar esta investigación fueron Liquozyme Supra (amilasa), Dextrozyme GA (glucoamilasa EC 3.2.1.3) y Promozyme D2 (pululanasa), de la marca comercial Novozymes y suministradas por la empresa COLDANZIMAS limitada de Bogotá, Colombia. [11].

\section{Hidrólisis enzimática}

El proceso de hidrólisis al cual se sometió al almidón de ñame, consistió en la aplicación de las 3 enzimas mencionadas anteriormente ( Liquozyme, dextroxyme y promozyme) en diferentes secuencias de aplicación; en el proceso de hidrólisis 1 se aplicó la secuencia Liquozyme - Promozyme - Dextrozyme $(L-P-D)$ y para el proceso de hidrólisis 2 se aplicó la secuencia Liquozyme - Dextrozyme - Promozyme $(L-D-P)$; en cada uno de los procesos se utilizaron soluciones al 36\% y al $46 \%$ de concentración de almidón de ñame. 
Proceso de hidrólisis 1

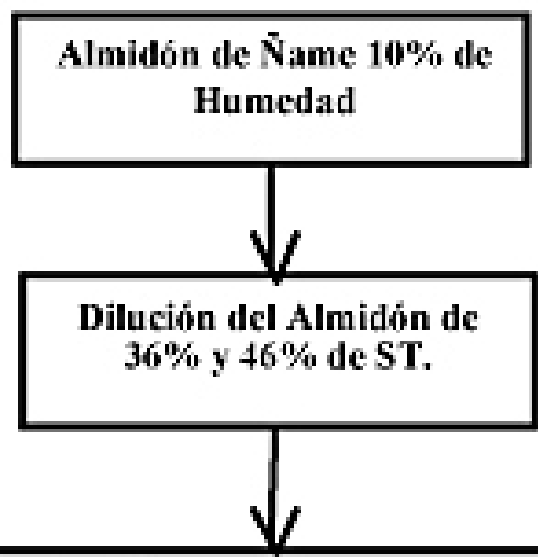

Aplicación de Liquozyme $0.052 \%$ /

peso seco del almidón. pH 5,4.

Licuefacción 1: $105^{\circ} \mathrm{C}-5 \mathrm{~min}$.

Licuefacción 2: $95^{\circ} \mathrm{C}-60 \mathrm{~min}$.

Proceso de hidrólisis 2

Aplicación de Destrozyme

Aplicación de Promozyme $0.06 \%$ / peso seco del $0.09 \%$ / peso seco del almidón. $\mathrm{pH} 5,2,58{ }^{\circ} \mathrm{C}-24$ almidón. $\mathrm{pH} 4,1,60^{\circ} \mathrm{C}-48$ haras

harae

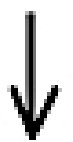

Aplicación de Destrozyme $0.06 \%$ / peso seco del almidón. $\mathrm{pH} 4,1.60{ }^{\circ} \mathrm{C}-48$ horas

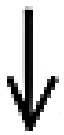

Aplicación de Promozyme $0.09 \%$ / peso seco del almidón. pH $5,2.58^{\circ} \mathrm{C}-24$ horas

Fuente: los autores

Figura 1. Procesos de hidrólisis aplicados al almidón de ñame.

Se tomaron cada una de las cantidades calculadas de almidón y agua en un matraz erlenmeyer de 1 litro, se agitaron hasta lograr la mezcla y total dilución del almidón; el pH fue ajustado a 5,4 con una solución de ácidoácido clorhídrico al 37\%, se adicionó 0,052\% de enzima liquozyme teniendo en cuenta el peso seco del almidón utilizado en la dilución en cada una de las concentraciones (el $36 \%$ y el $46 \%$ ) 
y se le puso un tapón de caucho con un termómetro adaptado por la mitad del tapón para controlar la temperatura de proceso.

La mezcla obtenida, almidón diluido más liquozyme, se sometió a un calentamiento progresivo con agitación constante en una estufa de agitación magnética marca termolyne hasta alcanzar $105^{\circ} \mathrm{C}$ y sosteniéndola por 5 minutos. Luego, se disminuyó rápidamente la temperatura hasta $95^{\circ} \mathrm{C}$ en un baño de agua a temperatura ambiente, se sostuvo a esta temperatura en la estufa con agitación constante por una hora hasta alcanzar la licuefacción completa, la cual se determinó por el cambio paulatino de estado pastoso a líquido.

Al jarabe producto de la licuefacción, en el proceso de hidrólisis 1 , se le redujo la temperatura hasta 58 ${ }^{\circ} \mathrm{C}$, se le ajustó el pH a 5,2 con solución de ácido clorhídrico al $37 \%$, se le adicionó el 0,09\% de enzima promozyme y se sostuvo en estas condiciones por 24 horas con agitación constante. En el proceso de hidrólisis 2, al jarabe obtenido en la licuefacción se le redujo la temperatura hasta $60^{\circ} \mathrm{C}$, se ajustó el pH a 4,1 con solución de ácido clorhídrico al $37 \%$, se le adicionó el $0,06 \%$ de la enzima dextrozyme y se sostuvo en estas condiciones por 48 horas.

El proceso de hidrólisis 1 continuó con la aplicación de la enzima dextrozyme y el proceso de hidrólisis 2 con la aplicación de la enzima promozyme en las condiciones mencionadas anteriormente para cada enzima. Luego de aplicar cada enzima se tomaron 30 gramos de muestra y se dividieron por triplicado en cada proceso; cada muestra fue identificada con un código y se procedió a realizar los análisis fisicoquímicos de grados Brix, según la norma [12], método [7]. pH nor- mas [10]; método [9]. Humedad por la norma [6]; método [7]. Azúcares totales, azúcares reductores y equivalente dextrosa por el método volumétrico o iodométrico de Lane Eynon modificado y adaptado de las normas [13]; método [7].

\section{Diseño estadístico experimental}

Para realizar esta investigación se aplicó un modelo factorial $2^{2}$. El objetivo fue estudiar el efecto del factor "tipo de proceso" de hidrólisis enzimática aplicado, y el factor "concentración de almidón" de ñame utilizado sobre la variable respuesta "el equivalente dextrosa" (ED) de cada jarabe obtenido luego de aplicar cada enzima en los respectivos procesos.

Para determinar el efecto de los factores principales y la interacción de los factores sobre el equivalente dextrosa del jarabe se procesaron los datos en el software statgraphics plus versión 5.1 y la hoja de calculo Excel de Microsoft office 2003.

\section{Resultados y Discusión}

\section{Obtención y caracterización del almidón de ñame}

La tabla 1 presenta los resultados obtenidos de la caracterización del almidón de ñame espino.

Tabla 1. Composición del almidón de ñame obtenido

\begin{tabular}{|c|c|}
\hline Anбlisis & Concentraciyn en \% \\
\hline Humedad & $10 \pm 0,39$ \\
\hline Proteína & $1,43 \pm 0,005$ \\
\hline Grasa & $0,046 \pm 0,0001$ \\
\hline Almidón ${ }^{*}$ & $79,64 \pm 0,12$ \\
\hline Almidón ${ }^{*}$ & $80,45 \pm 0,63$ \\
\hline Ceniza & $0,165 \pm 0,005$ \\
\hline Fibra & $0,7 \pm 0,05$ \\
\hline Amilosa & $40,75 \pm 1,01$ \\
\hline Amilopectina & $59,24 \pm 1,01$ \\
\hline \hline
\end{tabular}

Fuente: Ios autores.

*Cuantificado por espectrofotometría. **Según la norma COVENIN-376 (1981). 
Los valores obtenidos están acordes con los reportados por Dufour y col [14], excepto el con- tenido de amilosa, pues es mayor el encontrado en esta investigación $(40,75 \%)$ que el reportado por [1] de $31,1 \%$ y Freitas de $36 \%$ [15], en otras variedades de ñame y para otros tubérculos y cereales reportados por diferentes autores [16], [17], [18].

\section{Hidrólisis enzimática del almidón de ñame}

En las tablas 2 y 3 se pueden observar los valores de las medias y su desviación estándar para azúcares reductores, azúcares totales, equivalente dextrosa, sólidos, grados Brix y $\mathrm{pH}$, en cada uno de los procesos aplicados, variando las enzimas dextrozyme y promozyme en cada secuencia de enzima utilizada.

Tabla 2. Caracterización de los jarabes obtenidos en cada proceso de hidrólisis enzimática al utilizar solución de almidón de ñame al $36 \%$

\begin{tabular}{||l|c|c|c|c|c|c||}
\hline $\begin{array}{c}\text { Enzima } \\
\text { Aplicada }\end{array}$ & $\begin{array}{c}\% \text { Az. } \\
\text { Reductores }\end{array}$ & $\begin{array}{c}\% \text { Az. } \\
\text { Totales }\end{array}$ & $\begin{array}{c}\% \text { Eq. } \\
\text { Dextrosa }\end{array}$ & $\begin{array}{c}\% \text { Sylidos } \\
\text { Grados }\end{array}$ & pH \\
\hline Liq. & $6,87 \pm 0,28$ & $8,64 \pm 0,27$ & $18,82 \pm 0,83$ & $36,50 \pm 0,31$ & $34,47 \pm 0,12$ & $5,25 \pm 0,05$ \\
\hline Pro.-P1 & $12,75 \pm 0,85$ & $15,75 \pm 0,29$ & $34,15 \pm 1,90$ & $37,32 \pm 0,56$ & $35,2 \pm 0,28$ & $5,23 \pm 0,08$ \\
\hline Pro.-P2 & $30,23 \pm 0,35$ & $31,19 \pm 0,54$ & $77,39 \pm 2,61$ & $39,09 \pm 1,24$ & $37,3 \pm 0,46$ & $5,29 \pm 0,08$ \\
\hline Dex.-P1 & $27,42 \pm 0,37$ & $31,48 \pm 0,31$ & $68,97 \pm 1,20$ & $39,76 \pm 0,43$ & $36,2 \pm 0,60$ & $4,21 \pm 0,04$ \\
\hline Dex.-\%P2 & $22,13 \pm 0,72$ & $23,07 \pm 0,63$ & $59,44 \pm 2,01$ & $37,24 \pm 0,12$ & $34,73 \pm 0,31$ & $4,24 \pm 0,04$ \\
\hline
\end{tabular}

Liq.: Liquozyme; Pro.: Promozyme; Dex.: Dextrozyme.

Fuente: los autores.

Tabla 3. Caracterización de los jarabes obtenidos en cada proceso de hidrólisis enzimática al utilizar solución de almidón de ñame al $46 \%$

\begin{tabular}{|l|c|c|c|c|c|c|}
\hline \hline $\begin{array}{l}\text { Enzima } \\
\text { Aplicada }\end{array}$ & $\begin{array}{c}\% \text { Az. } \\
\text { Reductores }\end{array}$ & $\begin{array}{c}\text { Totales } \\
\text { Tz. }\end{array}$ & $\begin{array}{c}\text { \% Eq. } \\
\text { Dextrosa }\end{array}$ & & $\begin{array}{c}\text { Gylidos } \\
\text { Brix }\end{array}$ & \\
\hline Liq. & $10,54 \pm 1,33$ & $11,20 \pm 0,56$ & $22,15 \pm 2,77$ & $47,56 \pm 0,72$ & $42,6 \pm 0,20$ & $5,23 \pm 0,04$ \\
\hline Pro.-P1 & $18,59 \pm 0,46$ & $30,52 \pm 0,33$ & $38,58 \pm 0,73$ & $48,19 \pm 0,33$ & $43,4 \pm 0,20$ & $5,36 \pm 0,06$ \\
\hline Pro.-P2 & $52,05 \pm 0,82$ & $60,26 \pm 1,63$ & $78,99 \pm 1,77$ & $65,91 \pm 1,62$ & $60,20 \pm 04$ & $5,35 \pm 0,08$ \\
\hline Dex.-P1 & $50,92 \pm 0,88$ & $54,79 \pm 0,82$ & $75,54 \pm 1,48$ & $67,41 \pm 0,16$ & $58,00 \pm 0,20$ & $4,23 \pm 0,02$ \\
\hline Dex.-P2 & $43,88 \pm 0,47$ & $47,26 \pm 0,85$ & $67,76 \pm-1,77$ & $64,78 \pm 1,27$ & $56,27 \pm 0,42$ & $4,19 \pm 0,04$ \\
\hline
\end{tabular}

Liq.: Liquozyme; Pro.: Promozyme; Dex.: Dextrozyme.

Fuente: los autores.

Se observó una producción de los reductores desde un 6,87\% hasta un $52,05 \%$; los totales de un $8,64 \%$ a un $60,26 \%$. Se logró un equivalente dextrosa entre $18,82 \%$ y $78,99 \%$, los sólidos aumentaron del $35 \%$ al $70 \%$ y los grados Brix del $34 \%$ al $69 \%$. Los rangos anteriores se encuentran dentro de los reportados por diversos autores [19], [20], [21], que utilizaron almidón de plátano, yuca y papa.

En la figura 1 al igual que en las figuras 2, 3 y 4 se puede observar la variación analizada anteriormente para azucares reductores, azucares totales y equivalente dextrosa en cada uno de los procesos aplicados variando las enzimas dextrozyme y promozyme con cada concentración de almidón de ñame utilizado en cada secuencia de enzima utilizada. 
Proceso de hidrolisis enzimatica 1 con soluciòn de almidòn al $36 \%$

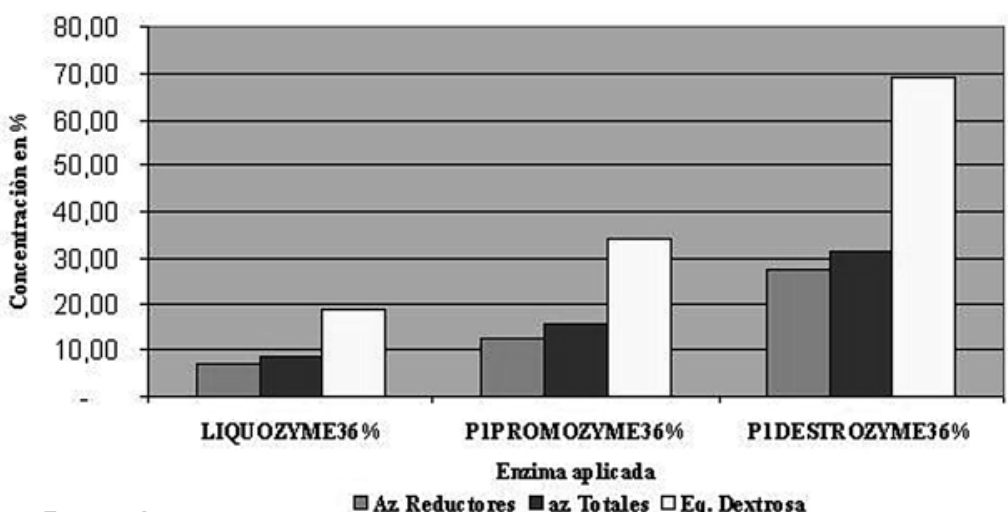

Fuente: los autores.

$\square$ Az Reductores $\square_{\text {az Totales }} \square \mathrm{Eq}$. Dextrosa

Figura 2. Resultados de la hidrólisis enzimática con la secuencia L-P-D utilizando solución de almidón al 36 \%.

Proceso de hidılisis enzimatica 2 con soluciòn de almidòn al 36\%

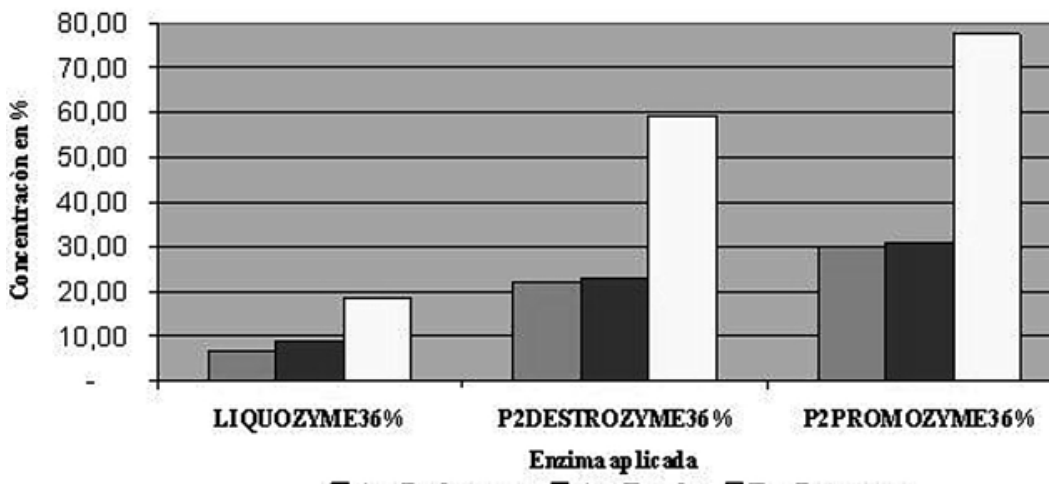

Fuente: los autores.

$\square \mathrm{Az}$. Reductores $\square \mathrm{Az}$. Totales $\square \mathrm{Eq}$. Dextrosa

Figura 3. Resultados de la hidrólisis enzimática con la secuencia L-D-P utilizando solución de almidón al $36 \%$.

Proceso de lửlólisis enzimatica 1 utilizando soluciòn de almidòn al $46 \%$

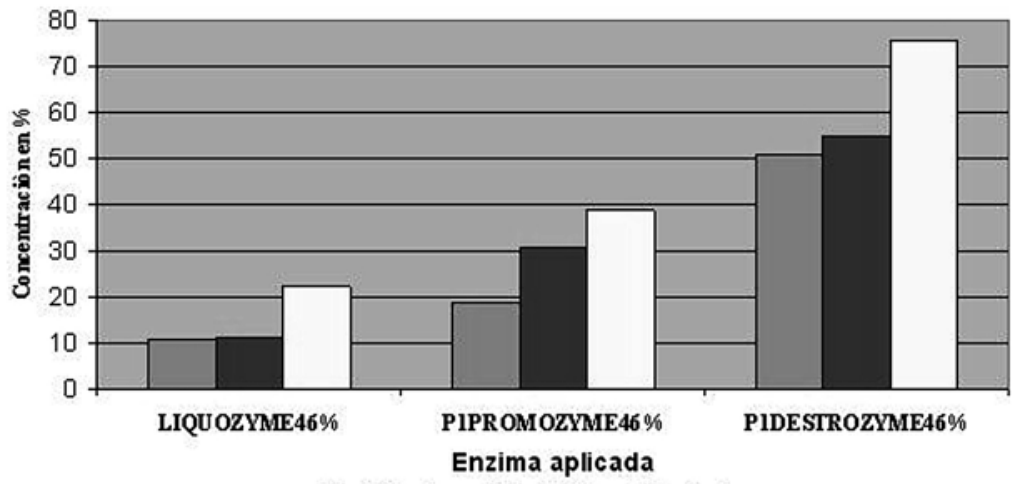

Fuente: los autores.

$\exists$ Az Reductores $\square$ Az Totales $\square \mathrm{Eq}$. Dextrosa

Figura 4. Resultados de la hidrólisis enzimática con la secuencia L-P-D utilizando solución de almidón al $46 \%$. 
Proceso de hidrolisis enzimatica 2 utilizando soluciòn de alimidòn al $46 \%$

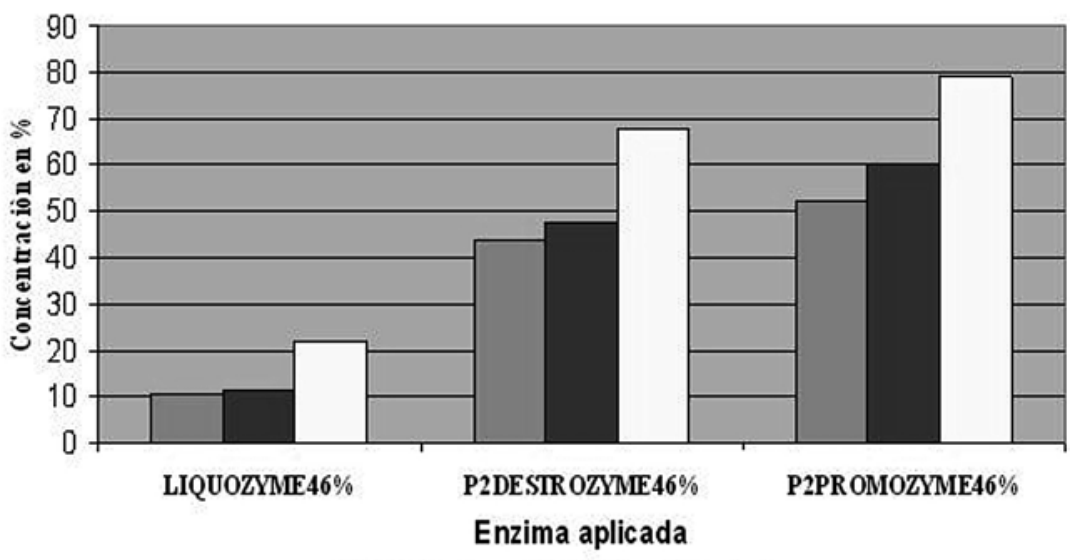

$\square_{\text {Az Reluc tores }} \boldsymbol{\nabla}_{\mathrm{Az}}$ Totales $\square_{\mathrm{Eq}}$. Dextrosa

Fuente: los autores.

Figura 5. Resultados de la hidrólisis enzimática con la secuencia L-D-P utilizando solución de almidón al $46 \%$.

\section{Licuefacción del almidón de ñame por medio de la aplicación del enzima liquozyme}

Del análisis de varianza y la prueba de comparación de medias de las menores diferencias significativas de Fisher (LSD) se determinó que al utilizar una concentración del $36 \%$ se obtiene $18,81 \%$ de equivalente dextrosa y para una concentración del 46\% de almidón se obtiene un 22,15\% (Ver tabla 4).

Tabla No 4. Resumen Estadístico para Equivalente Dextrosa de la licuefacción del almidón

\begin{tabular}{|l|l|l|l|l|}
\hline$\%$ de Almidón & Media & Mediana & Desviación típica & Error estándar \\
\hline 36 & 18,81 & 18,76 & 0,83 & 0,34 \\
\hline 46 & 22,15 & 22,85 & 2,77 & 1,13 \\
\hline Total & 20,48 & 19,70 & 2,62 & 0,76 \\
\hline
\end{tabular}

Fuente: elaboración propia.

En la tabla 5 se aprecia que hay diferencia significativa entre las medias del \% del Almidón; y que la mejor alternativa es la del $46 \%$.

Tabla 5. Contraste múltiple de rango para equivalente dextrosa según \% Almidón utilizado en la licuefacción

\begin{tabular}{||l|c|c|c||}
\hline \multicolumn{4}{||l||}{ Método: 95,0 \% LSD } \\
\hline \%Almidón & Frec. & Media & Grupos homogéneos \\
\hline 36 & 6 & 18,81 & X \\
\hline 46 & 6 & 22,15 & X \\
\hline Contraste & & & Diferencias +/- Límites \\
& & & \\
\hline $36-46$ & & ${ }^{\star}-3,34$ & 2,63 \\
\hline
\end{tabular}

* indica una diferencia significativa.

Fuente: los autores. 
Lo anterior permiten confirmar la actividad de la $\partial$ - amilasa liquozyme sobre el almidón de ñame y que la mejor alternativa es la del $46 \%$; el aumento de la concentración de almidón del $36 \%$ al $46 \%$ produce un incremento de solo 3,34 puntos en el equivalente dextrosa del jarabe; esto puede ser debido a que el aumento de la concentración de almidón disminuye la actividad de la $\partial$ - amilasa [22].

El equivalente dextrosa obtenido para las concentraciones del $36 \%$ y del 46 de almidón utilizado puede ser debido al alto contenido de amilosa encontrado en el almidón de ñame hidrolizado (un 40,75\%); teniendo en cuenta que para la hidrólisis enzimática del almidón de papa con un $26,9 \%$ de contenido de amilosa [23] y el 27\% para el maíz [16] se producen en la etapa de la licuefacción valores de equivalente dextrosa entre el $9 \%$ y el $15 \%$ al utilizar concentraciones de almidón entre el $20 \&$ y el $35 \%$. El incremento en la concentración de maltodextrinas en el proceso de licuefacción disminuye la actividad enzimática de las $\partial$ - amilasa, las maltodextrinas pueden ser hidrolizadas por la $\partial$ - amilasa y se presenta una competencia por sustrato [22].

Los productos obtenidos, se pueden considerar como jarabes intermedios de diferente composición ricos en oligosacáridos de cadenas lineales cortas o maltodextrinas que pueden ser más adelante, convertidos por enzimas específicas a otros productos como jarabes ricos en maltosa, jarabes de alto contenido de fermentables. Tambien en jarabes utilizados en la industria de la fabricación de helados, más por el aporte de sus propiedades físicas que por su poder edulcorante; jarabes de gran interés por sus propiedades funcionales como la capacidad de formar geles, pastas y la estabilización de emulsiones agua/aceite [24], [6].

\section{Sacarificación del almidón licuefaccionado por medio de la aplicación de promozyme}

A partir del análisis de varianza, el método de comparaciones de medias de Fisher (LSD) y los contrastes de hipótesis para los factores proceso y porcentaje de almidón se determinó que hay interacción entre el porcentaje de Almidón y el tipo de proceso, estos factores tienen efecto estadísticamente significativo sobre el equivalente dextrosa contenido en el jarabe. Se observa a su vez como el cambio de concentración de almidón afecta negativamente al equivalente dextrosa en los 2 procesos, acentuándose mucho más en el proceso 2 y disminuyendo el equivalente dextrosa del 17,95\% al 11,23\%, mientras que en el proceso 1 la disminución es más leve, del 15,08\% al 14,43\%. El factor proceso, no incide de forma aislada sobre el equivalente dextrosa del jarabe al no existir diferencia significativa entre las medias de tipo de proceso, lo cual se puede apreciar en la tabla 6.

Tabla 6. Análisis de la varianza para equivalente dextrosa para la aplicación de la enzima promozime

\begin{tabular}{|l|l|l|l|l|l|}
\hline Fuente & $\begin{array}{l}\text { Suma de } \\
\text { Cuadrados }\end{array}$ & GL & Cuadrado Medio & Cociente-F & P-Valor \\
\hline EFECTOS PRINCIPALES & & & & & \\
\hline A: \% Almidón & 40,68 & 1 & 40,68 & 8,22 & 0,0209 \\
\hline B: Proceso & 0,09 & 1 & 0,09 & 0,02 & 0,90 \\
\hline INTERACCIONES & & & & & \\
\hline AB & 27,75 & 1 & 27,75 & 5,61 & 0,0454 \\
\hline RESIDUOS & 39,58 & 8 & 4,95 & & \\
\hline TOTAL & 108,09 & 11 & & & \\
\hline
\end{tabular}

Los cocientes $F$ están basados en el error cuadrático medio residual. $F_{1,8 ; 0,05}=5.32$

Fuente: elaboración propia

Los resultados anteriores, indican que la reducción del contenido de agua por el efecto del aumento del contenido de sólidos (almidón) en la dilución inicial utilizada para la hidrólisis complica el desarrollo de la hidrólisis, lo que aumenta la viscosidad, y exige una mayor agitación y temperatura de hidrólisis 
o el cambio de proceso para lograr una adecuada hidrólisis [25]. El aporte obtenido de equivalente dextrosa en la aplicación de la enzima promozyme en el proceso de hidrólisis 1 (L-P-D) y en el proceso de hidrólisis 2 (L-D-P) con concentraciones del $36 \%$ y $46 \%$ de almidón se puede comprobar en las figuras $11,12,13$ y 14 . El producto obtenido en el proceso 1 , con un equivalente dextrosa acumulado del $34,34 \%$ y del $38,58 \%$ puede ser considerado un jarabe de baja conversión [26].

Los resultados anteriores permiten confirmar la acción de la enzima promozyme, como una pululanasa sobre el enlace $\partial-1,6$ de la amilopectina parcialmente hidrolizada en forma de maltotriosa en la etapa de la licuefacción y luego de la sacarificación parcial por parte la enzima dextrozyme como una solución al posible problema de la acumulación de isomaltosa por la acción especializada de la glucoamilasa dextrozyme sobre los enlaces alfa 1,4 y la hidrólisis lenta de los enlaces alfa 1,6 presentes en las maltodextrinas [27].

\section{Sacarificación del almidón licuefaccionado por medio de la aplicación de dextrozyme}

Tomando como base el análisis de varianza, el método de comparaciones de medias de Fisher (LSD) y los contrastes de hipótesis para los factores proceso y porcentaje de almidón, se determinó que no hay interacción entre los factores de concentración de almidón y el proceso que afecte el valor de equivalente dextrosa del jarabe. Estos factores tienen incidencia estadísticamente significativa de forma aislada sobre el valor del equivalente dextrosa del jarabe. Lo anterior toma como soporte el análisis de la tabla 7.

Tabla 7. Análisis de la Varianza para Equivalente Dextrosa para la aplicación de la enzima dextrozyme.

\begin{tabular}{|l|l|l|l|l|l|}
\hline Fuente & $\begin{array}{l}\text { Suma de } \\
\text { Cuadrados }\end{array}$ & GL & $\begin{array}{l}\text { Cuadrado } \\
\text { Medio }\end{array}$ & Cociente-F & P-Valor \\
\hline EFECTOS PRINCIPALES & & & & & \\
\hline A: \% Almidón & 40,68 & 1 & 40,68 & 8,22 & 0,0209 \\
\hline B: Proceso & 0,09 & 1 & 0,09 & 0,02 & 0,90 \\
\hline INTERACCIONES & & & & & \\
\hline AB & 27,75 & 1 & 27,75 & 5,61 & 0,0454 \\
\hline RESIDUOS & 39,58 & 8 & 4,95 & & \\
\hline TOTAL (CORREGIDO) & 108,09 & 11 & & & \\
\hline
\end{tabular}

Los cocientes $F$ están basados en el error cuadrático medio residual. $F_{1,}{ }_{8 ; 0,05}=5.32$

Fuente: elaboración propia

Los P-valores del porcetaje de almidón y proceso 0,0333 y 0,0013, comprueban la importancia estadística de cada uno de los factores de forma aislada. Dado que los p-valores de la concentración de almidón (\% almidón) y proceso son inferiores a 0,05 , estos factores tienen efecto estadísticamente significativo en el Equivalente Dextrosa para un 95,0\%; la figura 6 presenta claramente la no interacción de los factores del porcentaje de almidón y proceso. 


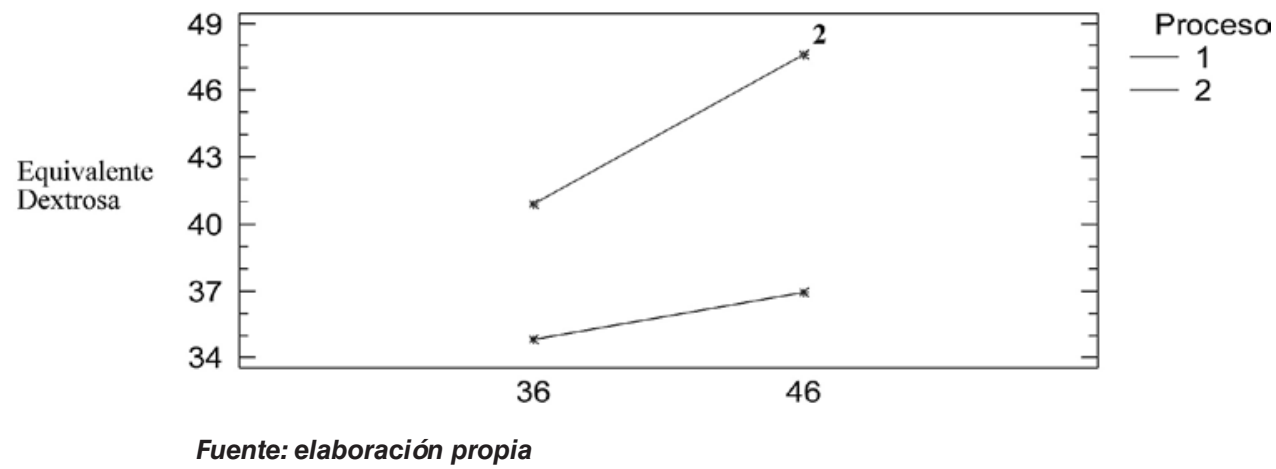

Figura 6. Efecto de la interacción del tipo de proceso y concentración de almidón sobre el equivalente dextrosa en la aplicación de la enzima dextrozyme.

En el proceso 1, se obtiene un incremento de 2,13 puntos en el equivalente dextrosa del jarabe al cambiar de una concentración del $36 \%$ al $46 \%$.

En el proceso 2, se obtiene el incremento de 6,71 puntos en el equivalente dextrosa del jarabe obtenido; tres veces más que en el proceso 1.

El mayor aporte al aumento del equivalente dextrosa del jarabe es logrado con la aplicación del proceso 2, en el cual se obtiene un equivalente dextrosa del $40,89 \%$ al $36 \%$ de concentración de almidón y aumenta hasta el $47,60 \%$ cuando se utiliza el $46 \%$ de almidón. La estimación del efecto almidón es del $4,42 \%$ y para el proceso es del $8,37 \%$.

Los resultados anteriores, indican que se obtienen mejores rendimientos de equivalente dextrosa en el jarabe a partir de la utilización del enzima destrozyme sobre una solución de almidón al $46 \%$ con la aplicación del proceso de hidrólisis 2 (liquozyme-dextrozyme-promozyme). Lo anterior, debido a que la hidrólisis enzimática se puede producir en procesos bajo condiciones de concentraciones de humedad reducida siempre y cuando el almidón sea completamente gelatinizado y fundido, lo que permite obtener jarabes de alto equivalente dextrosa [25]. Los valores obtenidos en el incremento del equivalente dextrosa, permiten confirmar la acción de la enzima dextroxime como una glucoamilasa sobre los enlaces $\partial-1,4$ y ว-1,6 del almidón licuado o parcialmente sacarificado [4], [25], [28], [29].

El incremento del equivalente dextrosa logrado en los procesos de hidrólisis aplicados son mayores que los reportados para el proceso de hidrólisis enzimática sobre el umari y para la yuca por Paredes y col., en 2001 y Grzeskowiak y col., en 2007, obtienen un incremento del equivalente dextrosa del 31\% al 33\% en la sacarificación del jarabe del almidón de papa en un reactor de ultrafiltración utilizando concentraciones del 32\% peso/peso de almidón [29], [30].

\section{Análisis del Jarabe final obtenido}

La tabla 8 presenta el análisis de la varianza para el equivalente dextrosa obtenido en el jarabe final luego de la aplicación del proceso de hidrólisis enzimática, esta permite determinar la existencia de interacción entre ambos factores; tanto la concentración de almidón utilizado y el tipo de proceso, son significativos para el equivalente dextrosa. El factor proceso es el que tiene mayor efecto sobre la media del equivalente dextrosa del jarabe; en el proceso 2 se obtiene un equivalente dextrosa mayor al utilizar la concentración del $36 \%$ y la del $46 \%$ de almidón. De igual manera, al aumentar la concentración de almidón de $36 \%$ al $46 \%$ se logra un aumento del equivalente dextrosa del jarabe en ambos procesos, pero de mayor valor en el proceso 2, el cual tiene un valor medio inicial de $77,39 \%$, hasta registrar su mayor concentración: 78,99\%.

Mientras que en el proceso 1 , se obtiene un valor de equivalente dextrosa inferior en ambas concentraciones de almidón utilizado, esto se puede observar en la figura 7. Lo anterior es debido a que dextrozyme es una amiloglucosidasa o glucoamilasa que actúa sobre los enlaces $\partial-1,4$ y $\partial-16$ de la amilosa y la amilopectina, mientras que promozyme es una pululanasa y tiene solo actividad sobre los enlaces $\partial-16$ de la amilopectina, pululano y dextrinas limites [16], [18]. 
Tabla 8. Análisis de la Varianza para Equivalente Dextrosa en el jarabe final

\begin{tabular}{|l|c|c|c|c|c||}
\hline Fuente & $\begin{array}{l}\text { Suma de } \\
\text { cuadrados }\end{array}$ & GL & $\begin{array}{l}\text { Cuadrado } \\
\text { Medio }\end{array}$ & Cociente-F & P-Valor \\
\hline EFECTOS PRINCIPALES & & & & & \\
\hline A: \% Almidón & 50,03 & 1 & 50,03 & 13,71 & 0,0050 \\
\hline B: Proceso & 105,82 & 1 & 105,82 & 31,12 & 0,0005 \\
\hline INTERACCIONES AB & 18,47 & 1 & 18,47 & 5,43 & 0,0481 \\
\hline RESIDUOS & 27,20 & 8 & 3,40 & & \\
\hline TOTAL (CORREGIDO) & 201,53 & 11 & & & \\
\hline
\end{tabular}

Los cocientes $F$ están basados en el error cuadrático medio residual. $F_{1},{ }_{8 ; 0,05}=5.32$

Fuente: elaboración propia

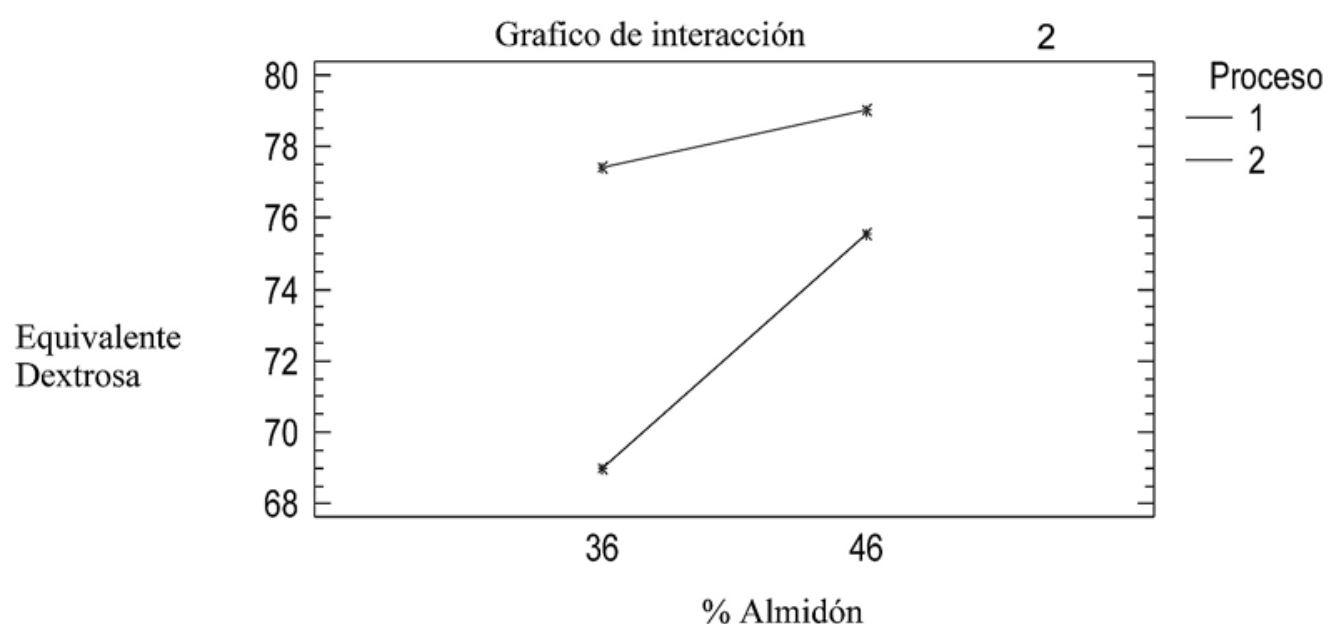

Fuente: elaboración propia

Figura 7. Efecto de la interacción del tipo de proceso y concentración de almidón sobre el equivalente dextrosa en el jarabe final.

Acorde con otros estudios, experimentalmente se ha demostrado que la hidrólisis del almidón a glucosa puede ser llevada a cabo en condiciones de concentración de sustrato superior al $65 \%$ y que los resultados obtenidos son específicos para cada tipo de glucoamilasa utilizada [27]. También se comprueba, que la estimación del efecto porciento Almidón es 4,08\% y la del efecto proceso es 5,94\%, lo que permite comprobar que el factor proceso es el de mayor incidencia en el equivalente dextrosa del jarabe; aunque la concentración de almidón también influye en el equivalente de dextrosa final del jarabe. Como se puede ver, la mejor alternativa es la de utilizar la concentración del $46 \%$ y el proceso 2 .

Los resultados obtenidos del equivalente dextrosa del jarabe final son superiores a los reportados para la hidrólisis de almidones de yuca (el 50\%) y batata (el 36\%) por Shariffa y col., en 2008 [21], pero inferior al obtenido a partir de la hidrólisis del almidón de plátano (un 80\%) por Her- nández et al., en 2008 [19]; para el almidón de maíz el 91\% obtenido por Van Der Veen et al., en 2006. Los jarabes obtenidos en esta investigación con equivalentes dextrosa del 78,99\% y del 77,39\%, se consideran de alta conversión por su alto contenido en glucosa [26],el valor del equivalente dextrosa obtenido en los jarabes durante la hidrólisis enzimática dependen del tiempo de reacción o incubación y la dosificación de enzima [27]. 


\section{Conclusiones}

El rendimiento obtenido en el procesamiento del ñame para la obtención de almidón y la composición del almidón, son acordes con lo reportado por diferentes autores, el contenido de amilosa encontrado en el almidón de ñame es superior.

En la etapa de licuefacción del almidón de ñame se produjeron jarabes intermedios que pueden ser utilizados por sus propiedades funcionales o como base para desarrollar otros procesos como el de la fermentación.

Al aplicar la pululanasa promozyme luego de la etapa de licuefacción se obtienen jarabes de baja conversión.

El equivalente dextrosa obtenido fue más significativo cuando se aplica dextrozyme luego de la etapa de licuefacción del almidón; es decir en el proceso 2 (L-D-P).

Se produjo un efecto altamente significativo de la concentración de almidón utilizada y el proceso de hidrólisis aplicado sobre el equivalente dextrosa del jarabe; el jarabe de mayor equivalente dextrosa se produce al utilizar una concentración de almidón del $46 \%$ aplicando el proceso de hidrólisis 2 (liquozymedextrozyme-promozyme).

El equivalente dextrosa del jarabe final obtenido de la hidrólisis enzimática del almidón de ñame variedad Dioscorea rotundata, permite clasificarlo como un jarabe de alta conversión.

El almidón de ñame variedad Dioscorea rotundata, puede ser utilizado como materia prima para obtener jarabes edulcorantes por medio de un proceso de hidrólisis enzimática.

La elección de una secuencia de aplicación de las enzimas para efectuar la hidrólisis enzimática del almidón de ñame, depende de las características necesarias de los jarabes a utilizar en la industria de alimentos.

\section{Referencias Bibliográficas}

[1] Corpoica \& Pronatta. (2003). Concepción de un modelo de agroindustria rural para la elaboración de harina y almidón a partir de raíces y tubérculos promisorios, con énfasis en los casos de achira (Canna edulis), arracacha (Arra- cacia xanthorriza) y ñame (Dioscorea sp.) Informe técnico final. $25-59$.

[2] FAO. (2003). Estadísticas de producción de cultivos a nivel mundial. Recuperado de http://apps. fao.org/page/form?collection=Production.Crops.Primary\&Domain=Production\&servlet=1\&languag e=ES \&hostname=apps.fao.org\&version=default.

[3] Colombia, Ministerio de Agricultura de Colombia. (2006). La agroindustria del azúcar en Colombia. Agrocadenas. Bogotá.

[4] Martínez Gallego Juan Francisco (2005). Utilización de alfa amilasas en la formulación de detergentes. (Tesis de Doctorado). Universidad de Granada. España.

[5] COVENIN (1981). Cacao y derivados. Norma 376: Determinación de Almidón. Caracas: EdicionesFondonorma.

[6] COVENIN (1994). Azucar. Norma 238: Determinación del contenido de humedad. Caracas: Ediciones Fondonorma.

[7] A.O.A.C. (2005). Oficial methods of a Análisis of the Associations of Oficial Analytical Chemists. 18 th. Cereal Food.

[8] McGrance, S.Jj., Cornell, H.J. \& Ris, C.J. (1998). A simple and rapid colorimetric method for the determination of amilose in starch products. Starch/Staerker. Citado en: Martinez Gallego, J.F. (2005). Utili- zación de alfa amilasas en la formulación de detergentes. (Tesis de Doctorado). Universidad de Granada. 
[9] A.O.A.C. 981.12E (1990). Official Methods of Analysis of the Association of Official Analytical Chemists. 15 ed. EEUU.

[10] COVENIN (1979). Alimentos. Norma 1315: Determinación de pH. Caracas: Ediciones Fondonorma.

[11] Novozymes. (2007). Fichas de aplicación de las enzimas Liquozyme, Promozyme y Dextrozyme suministradas por la empresa Coldanzimas. Bogota, Colombia.

[12] COVENIN (1983). Frutas y productos derivados. Norma 924: Determinación de sólidos solubles por refractometria. Cracas: Ediciones Fondonorma.

[13] COVENIN (1994). Azucar. Norma 3107: Determinación de azúcares reductores. Caracas: Ediciones Fondonorma.

[14] Dufour Dominique, Hurtado Jhon \& Gonzalo Rodríguez B. (2002). Procesamiento de dos especies de ñame (dioscorea alata, $d$. Rotundata): estudio de la factibilidad técnica y económica para la produc- ción de almidón y harina, CIAT, CORPOICA, CIRAC.

[15] Freitas, R. A., Paula, P. C., Feitosa, J. P. A., Rocha, S. \& Sierakwski M. R. (2004). Amylose contens, rheological properties and gelatinization kinetics of yam (Discorea alata) and Cassava (Manihot utilissi- ma) starches. Carbohydrate Polymers, Vol. 55, 3-8.

[16] Badui Salvador.(1993). Química de alimentos. (2 Ed.) México, Pearson educación.

[17] Dufour Dominique \& Alarcón Fredy. (2001). Almidón agrio de yuca en Colombia: planta procesadora: descripción y planos de equipos. Centro internacional de agricultura tropical, Cali-Colombia; centre de coopération internationale en recherche agronomique pour le développement, département daméliora- tion des méthodes pour l'innovation scientifique, Montpellier, Francia, 19-33.

[18] Fennema, Owen. (1993). Química de los alimentos. Ed.(2da ed). Acribia S.A. Segunda edición Zaragoza, España.

[19] Hernández Juan Pablo, Rodríguez Sandra \& Bello Luis. Obtención de jarabe fructosado a partir de almidón de plátano (Musa paradisíaca I.). (Tesis para obtener el grado de maestro). Instituto Politecnico Nacional, Mexico.

[20] Prochaskaa Krystyna, Kedzioraa Patrycja. (2007). Le Thanhb Joanna, Lewandowiczb Grazyna. Surrface Properties Of Enzymatic Hydrolysis Products Of Octenylsuccinate Starch Derivatives. Food Hy-drocolloids, 654-659.

[21] Shariffa Y.N., Karim A.A., Fazilah A., Zaiduly I.S.M. (2008) Enzymatic hydrolysis of granular native and mildly heat-treated tapioca and sweet potato starches at sub-gelatinization temperature, journal ho- mepage: Food Hydrocolloids, Vol. 23, 434-440.

[22] Baks Tim, Anja Janssen \& Remko M. Boom. (2006). The effect of carbohydrates on a-amylase activity measurements. Food and Bioprocess Engineering Group. Wageningen University and Research Center, P.O. Box 8129, 6700 EV, Wageningen, The Netherlands, Enzyme and Microbial Technology, Vol. 39, (2006) 114-119.

[23] Delgado R., Castro A.J. \& Vazquez A, M.(2009). A kinetic assessment of the enzymatic hydrolysis of potato (Solanum tuberosum). LWT - Food Science and Technology, Vol. 42, (2009) 797-804.

[24] Krzyzaniak Wojciech, Białas Wojciech, Olesienkiewicz Artur \& Jankowski Tomasz. (2003). Grajeky Włodzimierz, Characteristics of oligosaccharides produced By enzymatic hydrolysis of potato starch using mixture of pullulanases and alpha-amylases, Department of Biotechnology and Food Microbiology, The August Cieszkowski Agricultural University of Poznaz, Poland, Electronic Journal of Polish Agricultural Universities, Vol 6, cap.2, 16 - 25.

[25] Van der Veen M.E., Veelaert S. Van der Goot A.J. \& Boom R.M. (2006) Starch Hydrolysis Under Low Water Conditions. A Conceptual Process Design Journal of Food Engineering, Vol. 75, 178-186.

[26] Díaz García Armando, Zaldivar B Ricardo L \& Suárez Gricel. (2003). Análisis de la viscosidad de la glucosa cubana de la fábrica de hidrolizados de almidón de Cienfuegos. Universidad de Oriente, Haba- na, Cuba. Tecnología Química, Vol.23, No. 2. 
[27] Van Der Maarel Marc, Van der Veen Bart, Uitdehaag Joost C.M. \& Leemhuis Hans, Dijkhuizen L. (2002). Properties And Applications Of Starch-Converting Enzymes Of The _-Amylase Family. Journal of Biotechnology, Vol. 94, 137-155.

[28] Buchholz K., Seibel J. (2008). Industrial carbohydrate biotransformations, Carbohydrate Research. Vol 343, 1966-1979.

[29] Paredes Roger Ruiz. (2001). Hidrólisis enzimática de desechos del umarí (Poraqueiba sericea tulasne) y de la yuca (Manihot esculenta crantz). Revista Amazónica de Investigación Alimentaria, Vol.1, № 1, 22-29.

[30] Grzeskowiak-Przywecka Anna. (2007). Lucyna Słominska, Saccharification of potato starch in an ultrafiltration reactor. Journal of Food Engineering. Vol. 79, 539-545.

[31] Clarke M. A. \& SYRUPS (1993). Sugar Processing Research Institute Incorporated, New Orleans, LA, USA Encyclopaedia of Food Science, Food Technology and Nutrition. 5711- 5717.

[32] Official Methods of Analysis. (1990). Método AOAC 962.09, 1990. 15 th Edition. U.S.A.

[33] STATGRAPHICS. Plus para Windows 5.1. Edición profesional. [United States]: Statistical GraphicsCorp. 1999-2004. Numero de serie 53672201036640. 